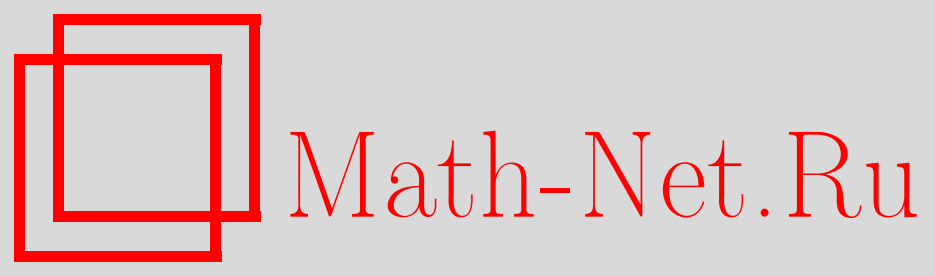

Ф. М. Малышев, Е. В. Кутырева, О распределении числа единиц в булевом треугольнике Паскаля, Дискрет. матем., 2006, том 18, выпуск 2, 123-131

DOI: https://doi.org/10.4213/dm51

Использование Общероссийского математического портала Math-Net.Ru подразумевает, что вы прочитали и согласны с пользовательским соглашением http://www . mathnet.ru/rus/agreement

Параметры загрузки:

IP: 54.80 .73 .141

26 апреля 2023 г., 15:11:17

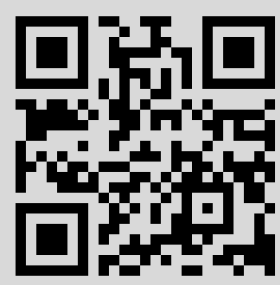


удк

\title{
О распределении числа единиц в булевом треугольнике Паскаля
}

\author{
() 2006 г. Ф. М. Малышев, Е. В. Кутырева
}

\begin{abstract}
Работа посвящена оценке числа булевых треугольников Паскаля достаточно большого размера $s$ с заданным числом единиц $\xi \leqslant k s, k>0$. Показано, что в любом подобном треугольнике Паскаля имеется нулевой треугольник, размер которого отличается от $s$ не более чем на константу, зависящую только от $k$. Доказано, что имеется монотонная неограниченная последовательность рациональных чисел $0=k_{0}<k_{1}<k_{2}<\ldots$ такая, что распределение числа треугольников сосредоточено в некоторых окрестностях точек $k_{i} s$. При этом вид распределения в каждой такой окрестности зависит не от $s$, а только от вычета $s$ по некоторому модулю, своему для каждого $i \geqslant 0$.
\end{abstract}

Рассматриваемые в работе обобщения треугольника Паскаля возникают, в частности, при исследовании клеточных автоматов. По этой тематике имеется обширная литература (см. [5-13]). Клеточный автомат использовался в качестве простой модели для изучения различных биологических процессов. Много работ посвящено изучению различных свойств симметрии треугольников Паскаля по некоторому модулю, генерируемых специальным классом клеточных автоматов (например, [2]). В [3] также изучаются некоторые свойства треугольников Паскаля, в частности, приведен способ нахождения числа специальных элементов некоторых строк треугольника Паскаля. С помощью клеточных автоматов можно строить схемы разделения секретов (см., например, [4]). Результаты данной работы касаются также двух следующих вопросов: какие статистические качества характеризуют поведение клеточного автомата и как шум и другие помехи воздействуют на клеточные автоматы?

Рассмотрим булев аналог треугольника Паскаля $T_{s}$, состоящий из $s$ строк элементов $G F(2)$. В этом треугольнике элементы $i$-й строки $x_{1}^{(i)}, x_{2}^{(i)}, \ldots, x_{i}^{(i)}, i=1, \ldots, s-1$, получаются из элементов $(i+1)$-й строки $x_{1}^{(i+1)}, x_{2}^{(i+1)}, \ldots, x_{i+1}^{(i+1)}$ по следующему правилу:

$$
x_{j}^{(i)}=x_{j}^{(i+1)} \oplus x_{j+1}^{(i+1)}, \quad j=1, \ldots, i .
$$

Последняя $s$-я строка $x_{1}^{(s)}, x_{2}^{(s)}, \ldots, x_{s}^{(s)}$ может быть произвольной. Число единичных элементов в треугольнике $T_{s}$ обозначим через $\xi$. Всего в треугольнике $T_{s}$ имеется $s(s+1) / 2$ элементов. Настоящая работа посвящена оценке числа треугольников $T_{s}$ (или, иначе, оценке числа $s$-х строк треугольников $T_{s}$ ) с заданным значением числа единиц $\xi \leqslant k s$ при достаточно больших $s$ и фиксированных $k>0$.

Через $s_{0}$ обозначим размер максимального треугольника $T_{s_{0}}$, состоящего целиком из нулевых элементов и содержащегося в треугольнике $T_{s}$. Предполагается, что каждая 


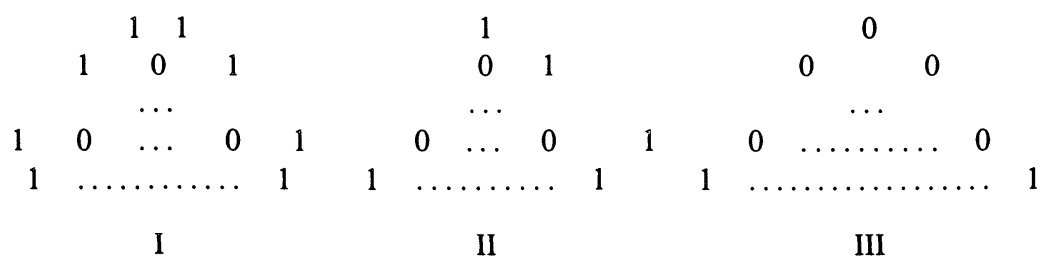

Рис. 1.

строка $T_{s_{0}}$ состоит из подряд идущих элементов одной из строк $T_{s}$. В общем случае нулевых треугольников $T_{s_{0}}$ в треугольнике $T_{s}$ с максимально возможным значением $s_{0}$ может быть несколько. Естественно, что величина $s_{0}$, как и конкретное расположение одного из треугольников $T_{s_{0}}$ в треугольнике $T_{s}$ зависят от конкретной $s$-й строки.

Нетрудно убедиться, что верно следующее утверждение.

Предложение 1. Для любого челого $R>0$ при достаточно больших $s>S$ в случае $s-s_{0} \leqslant R$ справедливо неравенство $\xi \leqslant k s$, где $k=k(R) u S=S(R)-$ зависящие от $R$ константы.

Справедливо и обратное к этому предложению утверждение.

Теорема 1. Для любого $k>0$ при достаточно больших $s>S$ в случае $\xi \leqslant k s$ справедливо неравенство $s-s_{0} \leqslant R$, где $R=R(k) u S=S(k)-$ зависячие от $k$ константы.

Доказательство. В приводимых ниже рассуждениях нам будет удобно элементы треугольника Паскаля приписывать вершинам одной из стандартных решеток евклидовой плоскости с углом в $60^{\circ}$ между ее образуюшими $e_{1}, e_{2}$ одинаковой длины 1 . Вершины с отметками находятся внутри и на границе правильного треугольника со стороной $s-1$. Сумма элементов булева треугольника Паскаля, приписанных к вершинам $v, v+e_{1}, v+e_{2}$, всегда нулевая. Вершины данной решетки являются пересечениями трех семейств параллельных прямых с образующими $e_{1}, e_{2}$ и $e_{2}-e_{1}$, соответственно. Соседние вершины с единичными отметками будем соединять отрезками этих прямых. Совокупность этих отрезков делит треугольник $T_{s}$ на фигуры трех типов, изображенных на рис. 1.

При этом нулевой треугольник, содержащийся в фигуре I-го типа, лежит строго внутри треугольника $T_{s}$, нулевой треугольник, содержащийся в фигуре II-го типа, касается одной из сторон треугольника $T_{s}$, а нулевой треугольник из фигуры III-го типа располагается при одной из вершин треугольника $T_{s}$. Фигуры типов II и III указаны с точностью до поворота на $120^{\circ}$. Обозначим через $t$ число нулей, лежащих на стороне нулевого треугольника каждой из этих фигур. Для фигур типов II и III $t \geqslant 1$, а для фигуры типа I $t \geqslant 0$. При $t=0$ шестиугольник типа I вырождается в треугольник без нулей с единицами при вершинах.

Пусть $\xi \leqslant k s$. Вначале докажем, что $s_{0} \geqslant c s$ для достаточно больших значений $s$, где $c=c(k)-$ зависящая от $k$ положительная константа. Чере $t_{1}, t_{2}, t_{3} \geqslant 0$ обозначим длины сторон фигур типа III. Положим $t_{i}=\alpha_{i}(s-1)$. Будем считать, что $0 \leqslant \alpha_{i} \leqslant 1 / 2$, $i=1,2,3$. Если, например, $\alpha_{1}>1 / 2$, то $s_{0} \geqslant \alpha_{1}(s-1)>(1 / 2)(s-1)>c s$ для любого $c<1 / 2$. Исключив из треугольника $T_{s}$ фигуры типа III с сохранением граничных вершин с единичными отметками, получим шестиугольник, который разбивается на фигуры типов I и II. 
Под площадью той или иной фигуры будем понимать площадь выпуклой оболочки ее вершин. За единицу площади возьмем площадь правильного треугольника $\left(T_{2}\right)$ со стороной 1 , равную $\sqrt{3} / 4$. Среднее число нулей на единицу площади в фигуре типа I равно

$$
\frac{t(t+1)}{2\left(t^{2}+4 t+1\right)}
$$

для фигуры типа II оно больше, именно, оно равно

$$
\frac{t(t+1)}{2\left(t^{2}+2 t\right)}=\frac{t+1}{2(t+2)}
$$

Обе эти величины растут с ростом $t$, поэтому число нулей на единицу площади шестиугольника, полученного из $T_{s}$ исключением фигур типа III, не превосходит

$$
\frac{s_{0}+1}{2\left(s_{0}+2\right)}=\frac{1}{2}\left(1-\frac{1}{s_{0}+2}\right) .
$$

Для числа нулей $N_{0}$ в шестиугольнике справедливо неравенство

$$
N_{0} \leqslant\left((s-1)^{2}-t_{1}^{2}-t_{2}^{2}-t_{3}^{2}\right) \frac{s_{0}+1}{2\left(s_{0}+2\right)},
$$

откуда для числа единиц в треугольнике $T_{s}$ получаем оценку

$$
\begin{aligned}
\xi \geqslant & \frac{s(s+1)}{2}-\frac{t_{1}\left(t_{1}+1\right)}{2}-\frac{t_{2}\left(t_{2}+1\right)}{2}-\frac{t_{3}\left(t_{3}+1\right)}{2}-\left((s-1)^{2}-t_{1}^{2}-t_{2}^{2}-t_{3}^{2}\right) \frac{s_{0}+1}{2\left(s_{0}+2\right)} \\
= & \frac{(s-1+1)(s-1+2)}{2}-\frac{(s-1) \alpha_{1}\left((s-1) \alpha_{1}+1\right)}{2}-\frac{(s-1) \alpha_{2}\left((s-1) \alpha_{2}+1\right)}{2} \\
& \quad-\frac{(s-1) \alpha_{3}\left((s-1) \alpha_{3}+1\right)}{2}-(s-1)^{2}\left(1-\alpha_{1}^{2}-\alpha_{2}^{2}-\alpha_{3}^{2}\right) \frac{1}{2}\left(1-\frac{1}{s_{0}+2}\right) \\
= & \frac{(s-1)^{2}}{2}\left(1-\alpha_{1}^{2}-\alpha_{2}^{2}-\alpha_{3}^{2}\right)+\frac{s-1}{2}\left(3-\alpha_{1}-\alpha_{2}-\alpha_{3}\right)+1 \\
& -\frac{(s-1)^{2}}{2}\left(1-\alpha_{1}^{2}-\alpha_{2}^{2}-\alpha_{3}^{2}\right)+\frac{(s-1)^{2}}{2}\left(1-\alpha_{1}^{2}-\alpha_{2}^{2}-\alpha_{3}^{2}\right) \frac{1}{s_{0}+2} \\
= & \frac{s-1}{2}\left(3-\alpha_{1}-\alpha_{2}-\alpha_{3}+\left(1-\alpha_{1}^{2}-\alpha_{2}^{2}-\alpha_{3}^{2}\right) \frac{s-1}{s_{0}+2}\right)+1 \\
\geqslant & \frac{s-1}{2}\left(\frac{3}{2}+\frac{1}{4} \frac{s-1}{s_{0}+2}\right) .
\end{aligned}
$$

У нас $\xi \leqslant k s \leqslant(k+\varepsilon)(s-1)$ при $s \geqslant k / \varepsilon+1$ для любого $\varepsilon>0$, поэтому

$$
\frac{s-1}{2}\left(\frac{3}{2}+\frac{1}{4} \frac{s-1}{s_{0}+2}\right) \leqslant(k+\varepsilon)(s-1) .
$$

Из последнего неравенства получаем, что

$$
s_{0} \geqslant \frac{s-1}{8\left(k+\varepsilon-\frac{3}{4}\right)}-2 \geqslant \frac{s}{8 k}
$$

при $s \geqslant(16 k(k+\varepsilon)-11 k) /(3 / 4-\varepsilon)$. В качестве требуемого $c$ можно взять, например, $c(k)=\min (1 /(8 k), 0,49)$. 


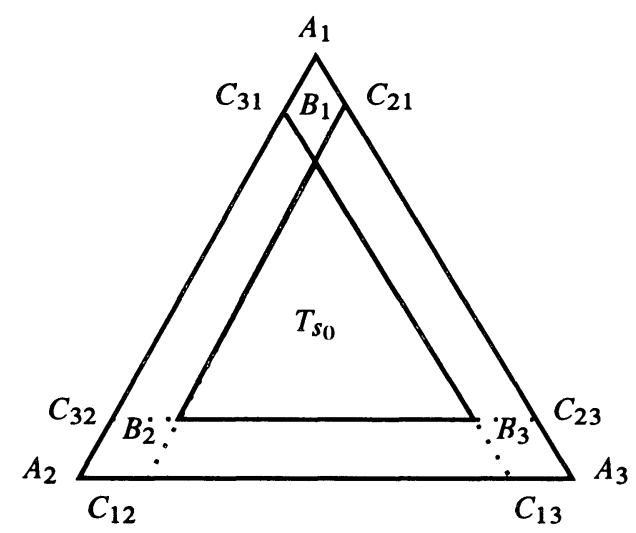

Рис. 2.

Обозначим далее чере $r_{1}, r_{2}, r_{3}$ числа строк между соответственными параллельными сторонами треугольников $T_{s}$ (с вершинами $\left.A_{1}, A_{2}, A_{3}\right)$ и $T_{s_{0}}$ (с вершинами $B_{1}, B_{2}, B_{3}$ ), увеличенные на 1 (см. рис. 2), обозначим также $r_{1}$ длину отрезка $\left[B_{2}, C_{12}\right], r_{2}$ длину отрезка $\left[C_{13}, A_{3}\right], r_{3}$ длину отрезка $\left[B_{1}, C_{31}\right]$. Для задания элементов, располагающихся на сторонах треугольника $T_{s}$, достаточно задать, например, значения элементов, находящихся на отрезках $\left[C_{12}, B_{2}\right),\left(B_{3}, C_{23}\right],\left[C_{31}, B_{1}\right)$. Число различных треугольников $T_{s} \mathrm{c}$ заданными значениями $r_{1}, r_{2}, r_{3}$ составляет, таким образом, $2^{\left[\left|r_{1}-1 / 2\right|\right]+\left[\left|r_{2}-1 / 2\right|\right]+\left[\left|r_{3}-1 / 2\right|\right]}$, (или $2^{\left(r_{1}-1\right)+\left(r_{2}-1\right)+\left(r_{3}-1\right)}$ при $\left.r_{i}>0, i=1,2,3\right)$.

Для завершения доказательства теоремы нужно проверить, что величины $r_{i}, i=1,2,3$, ограничены сверху зависящей от $k$ константой. Это будет следовать из того, что число единиц в трапеции $C_{12}, C_{13}, B_{3}, B_{2}$ (см. рис. 2) по порядку величины не меньше, чем $s_{0}\left(\log _{2} r_{1}\right) / 2$, где $s_{0} \geqslant c(k) s$. Докажем это.

Пусть вершины на полуинтервале $\left(B_{2}, C_{12}\right]$ имеют метки $x_{0}=1, x_{1}, \ldots, x_{r_{1}-1}$ (если бы $x_{0}=0$, то $s_{0}$ не было бы размером максимального нулевого треугольника в треугольнике $T_{s}$ ). Строку рассматриваемой трапеции, начинающуюся с элемента $x_{i}$, далее будем называть $i$-й строкой трапеции. Она ненулевая, так как в противном случае строка, первым элементом которой является $x_{0}$, также была бы нулевой ( $i$-я строка трапеции при $r_{1}>0$ находится на $\left(i+s-r_{1}+1\right)$-й строке треугольника $\left.T_{s}\right)$. Элементы $i$-й строки трапеции линейно выражаются через переменные $x_{0}, x_{1}, \ldots, x_{i}$, причем переменная $x_{i}$ на всех местах входит с коэффициентом 1 . Период $i$-й строки трапеции обозначим через $h(i)$.

Периодичность проверяется по индукции. Периодичность $i$-й строки следует из периодичности $(i-1)$-й строки, поскольку метки принимают значения из конечного поля $G F(2)$. Нулевая строка состоит сплошь из единиц.

Докажем, что первая строка имеет период 2 , две последующие имеют период $2^{2}$, у $2^{2}$ следующих период $2^{3}$ и так далее, то есть каждые $2^{i}$ следующих строк имеют период $2^{i+1}$, $i>0$. Поэтому число единиц в $i$-й группе строк будет не меньше, чем $\left[s_{0} / 2^{i+1}\right] 2^{i} \simeq s_{0} / 2$, $i \geqslant 0$, а число таких групп строк составляет около $\log _{2} r_{1}$. В результате для близкой к единице константы $c_{1}$ получаем, что

$$
k s \geqslant \xi \geqslant \frac{s_{0}}{2} c_{1} \log _{2} r_{1} \geqslant \frac{c_{1} c(k) s}{2} \log _{2} r_{1}
$$

откуда и следует ограниченность $r_{1}$. Аналогично, $r_{2}$ и $r_{3}$ ограничены. 


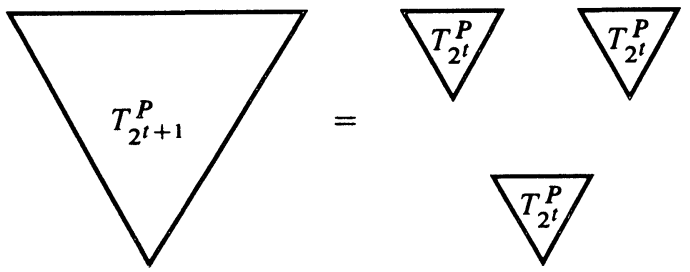

Рис. 3.

Использованное свойство периодичности достаточно проверить (в силу линейности) при $x_{1}=\ldots=x_{r_{1}-1}=0$, когда заполнение трапеции $C_{12}, C_{13}, B_{3}, B_{2}$ совпадает с частью специального булева треугольника Паскаля $T_{2^{t+1}-1}$, обозначаемой как $T_{2^{t}}^{P}$ и имеющей по $2^{t}$ единиц на каждой стороне (см. рис. 3). Здесь у треугольника Паскаля $T_{2^{t+1}-1}$ в последней строке только одна единица (в середине) и $2^{t+1}-2$ нулей, по $2^{t}-1$ нулей слева и справа. На $t$-м месте $i$-й строки трапеции находится элемент вида $x_{0} \alpha_{0, t}^{(i)}+x_{1} \alpha_{1, t}^{(i)}+\ldots+x_{i-1} \alpha_{i-1, t}^{(i)}+x_{i}, t \geqslant 0$. Требуемая периодичность последовательностей коэффищиентов $\left\{\alpha_{0, t}^{(i)}, t \geqslant 0\right\}, i \geqslant 0$, а значит, и последовательностей коэффициентов $\left\{\alpha_{j, t}^{(i)}, t \geqslant 0\right\}, i \geqslant j>0$, следует из симметричности треугольников $T_{2^{t}}^{\boldsymbol{P}}$, которые строятся в соответствии с рекурсивным равенством, приведенным на рис. 3 . На этом рисунке $t \geqslant 0$, $T_{1}^{P}=1$.

Теорема доказана.

Используемая в приведенном доказательстве техника позволяет при каждом $k>0$ вычислять все предельные точки множества $\{\xi / s \mid \xi \leqslant k s\}$. Величины $\xi / s$ данного множества отвечают тем треугольникам $T_{s}, s>0$, для которых $\xi \leqslant k s$ или (с некоторой погрешностью) $s-s_{0} \leqslant R=R(k)$.

При каждом $R$ число предельных точек конечно. Так, если $R \geqslant r_{1}+r_{2}+r_{3}=s-s_{0}$, причем $r_{1} \geqslant 0, r_{2} \geqslant 0, r_{3} \geqslant 0$ фиксированы, и для каждой из трех рассматриваемых выше трапеций заданы также значения $x_{0}=1, x_{1}, \ldots, x_{r-1} \in G F(2), r \in\left\{r_{1}, r_{2}, r_{3}\right\}$, то при каждом $s$ треугольник $T_{s}$ определяется однозначно, а предельной точкой будет $\chi=\lim _{s \rightarrow \infty} \xi / s$. Этот предел представляется суммой трех пределов по каждой трапеции отдельно: $\chi=\chi\left(r_{1}\right)+\chi\left(r_{2}\right)+\chi\left(r_{3}\right)$.

Из доказательства теоремы можно понять, что предел $\chi(r), r \in\left\{r_{1}, r_{2}, r_{3}\right\}$ (зависящий еще и от $x_{0}, x_{1}, \ldots, x_{r-1}$, хоть это и не отражено в обозначении $\chi(r)$ во избежание излишней громоздкости), равен числу единиц в матрице

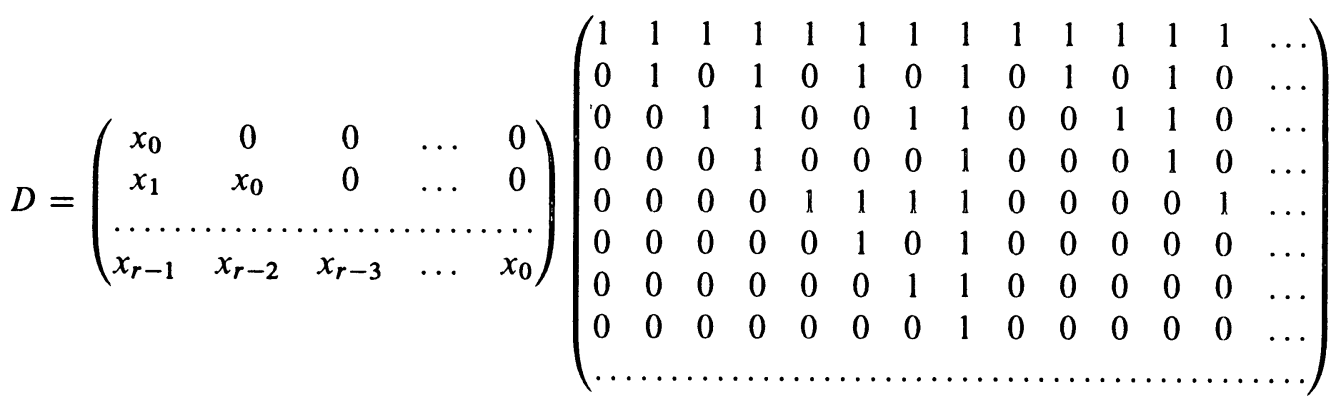

деленному на число столбцов в ней. Строка трапеции с номером $j=0,1, \ldots, r-1$ 
Таблица 1.

$$
\begin{aligned}
& \chi(0)=0 \\
& \chi(1)=1 ; \\
& \chi(2)=1 \frac{1}{2} \\
& \chi(3)=2 ; \\
& \chi(4)=2 \frac{1}{4}, 2 \frac{3}{4} ; \\
& \chi(5)=2 \frac{3}{4}, 3 \frac{1}{4} ; \\
& \chi(6)=3,3 \frac{1}{2}, 3 \frac{3}{4} ; \chi(7)=3 \frac{1}{4}, 3 \frac{3}{4}, 4,4 \frac{1}{4}, 4 \frac{1}{2} ; \chi(8)=3 \frac{3}{8}, 4 \frac{1}{8}, 4 \frac{3}{8}, 4 \frac{5}{8}, 4 \frac{7}{8}, 5 \frac{1}{8} ; \\
& \chi(9)=3 \frac{7}{8}, 4 \frac{5}{8}, 4 \frac{7}{8}, 5 \frac{1}{8}, 5 \frac{3}{8}, 5 \frac{5}{8} ; \\
& \chi(10)=4 \frac{1}{8}, 4 \frac{5}{8}, 4 \frac{7}{8}, 5 \frac{1}{8}, 5 \frac{1}{4}, 5 \frac{3}{8}, 5 \frac{1}{2}, 5 \frac{5}{8}, 5 \frac{3}{4}, 6,6 \frac{1}{4} ; \\
& \chi(11)=4 \frac{3}{8}, 4 \frac{7}{8}, 5 \frac{1}{8}, 5 \frac{3}{8}, 5 \frac{1}{2}, 5 \frac{5}{8}, 5 \frac{3}{4}, 5 \frac{7}{8}, 6,6 \frac{1}{8}, 6 \frac{1}{4}, 6 \frac{3}{8}, 6 \frac{1}{2}, 6 \frac{5}{8}, 6 \frac{3}{4} ; \\
& \chi(12)=4 \frac{1}{2}, 5 \frac{1}{4}, 5 \frac{1}{2}, 5 \frac{5}{8}, 5 \frac{3}{4}, 5 \frac{7}{8}, 6,6 \frac{1}{8}, 6 \frac{1}{4}, 6 \frac{3}{8}, 6 \frac{1}{2}, 6 \frac{5}{8}, 6 \frac{3}{4}, 6 \frac{7}{8}, 7,7 \frac{1}{8}, 7 \frac{1}{4} .
\end{aligned}
$$

отвечает $(j+1)$-й строке матрищы $D$. Здесь в матрице второго сомножителя $r$ строк и $2^{i}$ столбцов, $i=\left\lceil\log _{2} r\right\rceil$, над ее главной диагональю располагается треугольник $T_{2}^{P}$, деформированный так, что три угла по $60^{\circ}$ превратились, соответственно, в углы по $45^{\circ}$, $45^{\circ}$ и $90^{\circ}$. Период последней $(r-1)$-й строки трапеции равен в точности $2^{i}$.

Возможные значения $\chi(r)$ следует вычислять последовательно для $r \geqslant 1$, при этом каждый раз необходимо отслеживать число единиц на периоде $2^{\left\lceil\log _{2} r\right\rceil}$ только в последней строке матрицы $D$, отдельно при $x_{r-1}=0$ и при $x_{r-1}=1$.

На этом пути с помощью вычислительной техники были получены значения $\chi(r)$ для значений $r \leqslant 28$. Здесь, с целью экономии места, мы приведем их для $r \leqslant 12$ в табл. 1 .

Основная цель работы, как это было указано в ее начале, состоит в определении числа треугольников $T_{s}$ с числом единиц в нем $\xi \leqslant k s$, которое будем обозначать через $M_{s}(\xi)$. Величина $\xi$ может принимать, вообще говоря, целочисленные значения от нуля до величины порядка $s^{2} / 3$. Последняя величина достигается, например, для $\left(x_{1}^{(s)}, x_{2}^{(s)}, \ldots, x_{s}^{(s)}\right)=(1,0,1,1,0,1,1,0, \ldots)$. Бо́льшие значения $\xi$ принимать не может, поскольку вершины треугольника $T_{s}$ содержатся среди объединения трехточечных подмножеств $\left\{n\left(e_{1}+e_{2}\right)+m_{3} e_{1}, e_{1}+n\left(e_{1}+e_{2}\right)+m_{3} e_{1}, e_{2}+n\left(e_{1}+e_{2}\right)+m_{3} e_{1}\right\}, n, m \in \mathbf{Z}, \mathbf{\kappa}$ вершинам которых может быть приписано не более двух единиц, а число таких подмножеств, пересекающихся с $T_{s}$, имеет порядок $s^{2} / 6$, в чем убеждаемся, например, делением площади треугольника $T_{s}$ на площадь параллелограмма с вершинами $0, e_{1}+e_{2}, 3 e_{1}$, $4 e_{1}+e_{2}$.

Приведенные после доказательства теоремы рассуждения существенно уточняют область значений $\xi$ при $\xi \leqslant k s, k \geqslant 0$.

Следствие 1. Имеются монотонная неограниченная последовательность рачиональных чисел $0=k_{0}<k_{1}<k_{2}<\ldots$ и последовательность неотричательных чисел $\varepsilon_{i}, i \geqslant 0$, такие, что для любого фиксированного $k>0$ nрu $\xi \leqslant k s$ и достаточно больших $s>S(k)$ справедливо равенство $M_{s}(\xi)=0$, если $\xi \notin \bigcup_{i=0}^{\infty}\left(k_{i} s-\varepsilon_{i}, k_{i} s+\varepsilon_{i}\right)$.

Доказательство. Из приведенных выше рассуждений, следующих за доказательством теоремы, заключаем, что для каждого $k>0$ и при любом достаточно большом $s$ число треугольников $T_{s} \mathrm{c} \xi \leqslant k s$ не зависит от $s$, а зависит только от $k$. Каждый такой треугольник задается наборами целых неотрицательных чисел $r_{1}, r_{2}, r_{3}, r_{1}+r_{2}+r_{3}=s-s_{0} \leqslant R(k)$, и булевыми значениями $x_{i 0}=1, x_{i 1}, \ldots, x_{i, r_{i}-1}, i=1,2,3$. Последние параметры при $r_{i}=0$ отсутствуют. Зафиксировав $r_{1}, r_{2}, r_{3}$ и $x_{i j}, j=0,1, \ldots, r_{i}-1, i=1,2,3$, 
получаем серию однозначно задаваемых треугольников $T_{s}$, параметризованных величиной $s$, для которых $\lim _{s \rightarrow \infty} \xi / s=\chi\left(r_{1}\right)+\chi\left(r_{2}\right)+\chi\left(r_{3}\right)$. Тем самым, в качестве $k_{i}$, $i \geqslant 0$, выступает сумма любых трех чисел вида $\chi(r)$. Среди этих трех чисел могут быть и одинаковые. Если $i \geqslant 0$ зафиксировано и $k_{i}=\chi\left(r_{1}\right)+\chi\left(r_{2}\right)+\chi\left(r_{3}\right)$, то величина $\left(\chi\left(r_{1}\right)+\chi\left(r_{2}\right)+\chi\left(r_{3}\right)\right) s_{0}=\left(\chi\left(r_{1}\right)+\chi\left(r_{2}\right)+\chi\left(r_{3}\right)\right)\left(s-r_{1}-r_{2}-r_{3}\right)$ отличается от $\xi$ не более, чем на зависящую от $i$ константу. Отличие обусловлено единицами, не вошедшими в матрицы $D$ и приписанными к вершинам, содержащимся в четырехугольниках $A_{1} C_{21} B_{1} C_{31}, A_{3} C_{13} B_{3} C_{23}, A_{2} C_{32} B_{2} C_{12}$ (см. рис. 2). Для доказательства следствия остается заметить, что при каждом конкретном $k_{i}$ число возможностей для $r_{1}, r_{2}, r_{3}$ и $x_{j 0}=1, x_{j 1}, \ldots, x_{j, r_{j}-1}, j=1,2,3$, ограничено.

В силу приведенного следствия при $\xi \leqslant k s$ и достаточно больших $s$ обязательно $\xi \in \bigcup_{i: k_{i} \leqslant k}\left(k_{i} s-\varepsilon_{i}, k_{i} s+\varepsilon_{i}\right)$. Введем обозначение

$$
N_{i}=\sum_{\xi \in\left(k_{i} s-\varepsilon_{i}, k_{i} s+\varepsilon_{i}\right)} M_{s}(\xi) .
$$

Начиная с некоторых достаточно больших значений эта величина, как уже отмечалось, не зависит от $s$.

Ниже приводим первые 45 значений $k_{i}, i=0,1, \ldots, 44$, указывая в скобках соответствующие величины $N_{i}: 0(1), 1(3), 1 \frac{1}{2}(6), 2(15), 2 \frac{1}{4}(12), 2 \frac{1}{2}(12), 2 \frac{3}{4}(36), 3(61), 3 \frac{1}{4}(72)$, $3 \frac{3}{8}(24), 3 \frac{1}{2}(78), 3 \frac{3}{4}(120), 3 \frac{7}{8}(48), 4(216), 4 \frac{1}{8}(96), 4 \frac{1}{4}(204), 4 \frac{3}{8}(240), 4 \frac{1}{2}(368), 4 \frac{5}{8}(264)$, $4 \frac{3}{4}(240), 4 \frac{7}{8}(744), 5(660), 5 \frac{1}{16}(48), 5 \frac{1}{8}(600), 5 \frac{1}{4}(1128), 5 \frac{3}{8}(1440), 5 \frac{1}{2}(1560), 5 \frac{9}{16}(96)$, $5 \frac{5}{8}(1344), 5 \frac{3}{4}(2472), 5 \frac{13}{16}(96), 5 \frac{7}{8}(1896), 5 \frac{15}{16}(48), 6(4336), 6 \frac{1}{16}(192), 6 \frac{1}{8}(2712), 6 \frac{3}{16}(192)$, $6 \frac{1}{4}(5952), 6 \frac{5}{16}(144), 6 \frac{3}{8}(5376), 6 \frac{7}{16}(192), 6 \frac{1}{2}(9360), 6 \frac{9}{16}(1440), 6 \frac{5}{8}(5064), 6 \frac{11}{16}(624)$.

Сами слагаемые $M_{s}(\xi)$, упорядоченные по $\xi \in\left(k_{i} s-\varepsilon_{i}, k_{i} s+\varepsilon_{i}\right)$, в сумме

$$
N_{i}=\sum_{\xi \in\left(k_{i} s-\varepsilon_{i}, k_{i} s+\varepsilon_{i}\right)} M_{s}(\xi),
$$

вообще говоря, зависят от $s$, но они одинаковы для тех $s>S\left(k_{i}\right)$, у которых одинаковы вычеты по модулю $2^{\rho_{i}}, i>0$, где

$$
\rho_{i}=\max _{r_{1}, r_{2}, r_{3} \geqslant 0: \chi\left(r_{1}\right)+\chi\left(r_{2}\right)+\chi\left(r_{3}\right)=k_{i}} \max _{j=1,2,3}\left\lceil\log _{2} r_{j}\right\rceil \text {. }
$$

Проверим это. Если

$$
s_{0}+1=s-r_{1}-r_{2}-r_{3}+1=\alpha 2^{\rho_{i}}+\Delta, \quad \Delta \in\left\{0,1, \ldots, 2^{\rho_{i}}-1\right\}, \quad \alpha \in \mathbf{N},
$$

To

$$
\xi=\alpha 2^{\rho_{i}} k_{i}+f_{r_{1}, r_{2}, r_{3} ; x}(\Delta)
$$

Здесь $f_{r_{1}, r_{2}, r_{3} ; x}:\left\{0,1, \ldots, 2^{\rho_{i}}-1\right\} \rightarrow \mathbf{Z}$ - некоторое отображение, зависящее, кроме $i>0$, как от $r_{1}, r_{2}, r_{3}$, так и от значений $\left(x_{j 0}, x_{j 1}, \ldots, x_{j, r_{j}-1}\right) \in G F\left(2^{r_{j}}\right), j=1,2,3$, своих для каждой из трех трапеций.

Пусть $r_{1 i} \geqslant 0, r_{2 i} \geqslant 0, r_{3 i} \geqslant 0$ - один специально выделенный набор, для которого $\chi\left(r_{1 i}\right)+\chi\left(r_{2 i}\right)+\chi\left(r_{3 i}\right)=k_{i}$, и $s_{0}+1=s-r_{1 i}-r_{2 i}-r_{3 i}+1=\alpha_{1} 2^{\rho_{i}}+\Delta_{i}$, $\Delta_{i} \in\left\{0,1, \ldots, 2^{\rho_{i}}-1\right\}$. Величина $\Delta_{i}$ определяется по вычету $s\left(\bmod 2^{\rho_{i}}\right)$. 
Вместо $\xi=\xi_{s}$ рассмотрим центрированную величину

$$
\begin{aligned}
\zeta_{i} & =\zeta_{i, s}=\xi-\left(s-r_{1 i}-r_{2 i}-r_{3 i}-\Delta_{i}+1\right) k_{i}=f_{r_{1 i}, r_{2 i}, r_{3 i} ; x}\left(\Delta_{i}\right) \\
& \in\left(\left(r_{1 i}+r_{2 i}+r_{3 i}+\Delta_{i}-1\right) k_{i}-\varepsilon_{i},\left(r_{1 i}+r_{2 i}+r_{3 i}+\Delta_{i}-1\right) k_{i}+\varepsilon_{i}\right) .
\end{aligned}
$$

Для величины $M_{s}(\xi)$ введем новое обозначение

$$
m_{s}\left(\zeta_{i}\right)=M_{s}\left(\zeta_{i}+\left(s-r_{1 i}-r_{2 i}-r_{3 i}-\Delta_{i}+1\right) k_{i}\right)=M_{s}(\xi)
$$

Предложение 2. Если размеры двух треугольников $T_{s_{1}}, T_{s_{2}}$ являются достаточно большими, $s_{1}, s_{2}>S\left(k_{i}\right), u \xi_{s_{1}} \in\left(k_{i} s_{i}-\varepsilon_{i}, k_{i} s_{1}+\varepsilon_{i}\right), \xi_{s_{2}} \in\left(k_{i} s_{2}-\varepsilon_{i}, k_{i} s_{2}+\varepsilon_{i}\right)$, причем $s_{1} \equiv s_{2}\left(\bmod 2^{\rho_{i}}\right), \operatorname{mo} m_{s_{1}}\left(\zeta_{i}\right)=m_{s_{2}}\left(\zeta_{i}\right)$.

Предложение 2 следует из того, что для любых $r_{1} \geqslant 0, r_{2} \geqslant 0, r_{3} \geqslant 0$ таких, что $\chi\left(r_{1}\right)+\chi\left(r_{2}\right)+\chi\left(r_{3}\right)=k_{i}$, величина

$$
\begin{aligned}
\zeta_{i}=\xi- & \left(s-r_{1 i}-r_{2 i}-r_{3 i}-\Delta_{i}+1\right) k_{i}=\left(s-r_{1}-r_{2}-r_{3}-\Delta+1\right) k_{i} \\
& \quad+f_{r_{1}, r_{2}, r_{3} ; x}(\Delta)-\left(s-r_{1 i}-r_{2 i}-r_{3 i}-\Delta_{i}+1\right) k_{i} \\
= & f_{r_{1}, r_{2}, r_{3} ; x}(\Delta)+\left(r_{1 i}+r_{2 i}+r_{3 i}+\Delta_{i}\right) k_{i}-\left(r_{1}+r_{2}+r_{3}+\Delta\right) k_{i}
\end{aligned}
$$

не зависит от конкретного достаточно большого $s$ с заданным вычетом по модулю $2^{\rho_{i}}$, который определяет величины $\Delta$ и $\Delta_{i}$.

Предложение 2 устанавливает, что при каждом $i \geqslant 0$ две целочисленные случайные величины $\zeta_{i, s_{1}}$ и $\zeta_{i, s_{2}}$, определенные на множестве мощности $N_{i}$ с равномерным распределением вероятностей, имеют одинаковое распределение, если $s_{1}, s_{2}$ достаточно большие и $s_{1} \equiv s_{2}\left(\bmod 2^{\rho_{i}}\right)$. В этом случае случайные величины $\xi_{s_{1}}-k_{i} s_{1}$ и $\xi_{s_{2}}-k_{i} s_{2}$ тоже одинаково распределены, только не являются целочисленными.

Суммируя вышесказанное, можно заключить, что имеется строго возрастающая неограниченная последовательность рациональных чисел $\left\{k_{i}, i \geqslant 0\right\}$ такая, что при любом $k>0, k \notin\left\{k_{i}, i \geqslant 0\right\}$, для достаточно больших $s$ число $N(k)$ булевых треугольников Паскаля $T_{s}$ с числом единиц в нем $\xi_{s}<k s$ не зависит от $s$, а сами значения $\xi_{s}$ сосредоточены около величин $k_{i} s, k_{i}<k$, точнее, $\xi_{s} \in \bigcup_{i \geqslant 0: k_{i}<k}\left(k_{i} s-\varepsilon_{i}, k_{i} s+\varepsilon_{i}\right)$, где $\varepsilon_{i}, i \geqslant 0$, независимые от $s$ конечные величины. В результате, при каждом $i \geqslant 0$ с $k_{i}<k$ число $N_{i}$ треугольников $T_{s} \mathrm{c} \xi_{s} \in\left(k_{i} s-\varepsilon_{i}, k_{i} s+\varepsilon_{i}\right)$ тоже не зависит от $s, \sum_{i \geqslant 0: k_{i}<k} N_{i}=N(k)$. Распределение случайной величины $\xi_{s}$ при условии, что $\xi_{s} \in\left(k_{i} s-\varepsilon_{i}, k_{i} s+\varepsilon_{i}\right)$, зависит только от $i$ и от вычета $s\left(\bmod 2^{\rho_{i}}\right)$, где $\rho_{i}, i \geqslant 0,-$ неотрицательные целые числа. Заметим еще, что $\xi_{s}$ не может превосходить величины порядка $s^{2} / 3$, а число всех треугольников $T_{s}$ равно $2^{s}$.

\section{Список литературы}

1. Малышев Ф. М., Кутырева Е. В., Об одном свойстве булевых аналогов треугольника Паскаля. Обозрение прикладпой и промышлепной математики (2004) 11, №2, 245-246.

2. Barbe A., Symmetric patterns in the cellular automaton that generates Pascal's triangle modulo 2. Discrete Appl. Math. (2000) 105, 1-38.

3. Crespo C., Ponteville Ch., de Spinadel V. W., Divisibility cellular automata. Chaos Solitons Fractals (1995) 6, 105-112.

4. Marañon G. Á., Encinas L. H., del Rey Á. M., Sharing secret color images using cellular automata with memory. http://arxiv.org/abs/cs/0312034, 2003. 
5. Wolfram S., Cellular automata as simple self-organizing systems. Caltech preprint CALT-68-938, 1982.

6. Wolfram S., Cellular automata, Los Alamos Sci. (1983) 9, 2-21.

7. Wolfram S., Statistical mechanics of cellular automata. Rev. Mod. Phys. (1983) 55, 601-644.

8. Wolfram S., Universality and complexity in cellular automata. Physica (1984) 10D, 1-35.

9. Wolfram S., Martin O., Odlyzko A. M., Algebraic properties of cellular automata. Comm. Math. Phys. (1984) 93, 219-258.

10. Wolfram S., Computation theory of cellular automata, Comm. Math. Phys. (1984) 96, 15-57.

11. Wolfram S., Twenty problems in the theory of cellular automata. Physica Scripta (1985) T9, 170-183.

12. Wolfram S., Theory and Applications of Cellular Automata. World Scientific, Singapore, 1986.

13. Wolfram S., Cryptography with cellular automata. Lecture Notes Computer Sci. (1986) 218, 429-432.

Статья поступила 06.10.2004.

Переработанный вариант поступил 14.12.2005. 